\title{
Don't stop just yet! A simple, effective, and socially responsible approach to bus-stop consolidation
}

\author{
Colin Stewart \\ School of Urban Planning \\ McGill University \\ Suite 400, 815 Sherbrooke St. W. \\ Montréal, Québec, H3A 2K6 \\ Canada \\ Tel.: 514-398-4058 \\ Fax: 514-398-8376 \\ E-mail: colin.stewart2@mail.mcgill.ca
}

\author{
Ahmed El-Geneidy \\ School of Urban Planning \\ McGill University \\ Suite 400, 815 Sherbrooke St. W. \\ Montréal, Québec, H3A 2K6 \\ Canada \\ Tel.: 514-398-8741 \\ Fax: 514-398-8376 \\ E-mail: ahmed.elgeneidy@mcgill.ca
}

\begin{abstract}
August 2015
For citation please use : Stewart, C., \& El-Geneidy, A. (2016). Don't stop just yet! A simple, effective, and socially responsible approach to bus-stop consolidation. Public Transport: Planning and Operations, $8(1), 1-23$.
\end{abstract}




\begin{abstract}
Bus-stop consolidation is one of the most cost-effective ways for a transit agency to improve the quality of their bus services. By removing unnecessary stops, buses will have reduced runtimes, which can lead to higher frequencies and/or fewer buses on a route. Unfortunately, current research on bus stop consolidation and stop spacing focuses on complex mathematical models that are difficult for agencies to apply, and that overlook many important real-world considerations. The goal of this paper is to propose a new bus stop consolidation methodology that is realistic, simple, and effective, while at the same time being sensitive to people with reduced mobility and adaptable to the needs of different agencies. The new methodology is tested on the bus network of the Société de transport de Montreal (STM). Adopting this simple methodology is expected to remove $23 \%$ of the network's stops while only reducing the system coverage area by $1 \%$. The removal of these stops could result in morning-peak savings of 109 hours of operating time and the elimination of a bus from up to 75 routes at the system level. This methodology can be applied to any urban bus network, and thus can be of interest to transit agencies and transportation researchers.
\end{abstract}

Keywords: public transit, bus operations, bus stop consolidation, stop spacing, service coverage, walking distance 


\section{INTRODUCTION}

North American bus networks typically prioritize short walking distances at the expense of service quality (El-Geneidy, 2006). The mindset behind this is to provide an experience where passengers do not have to walk much more than they would if they were driving a car. However, this requires bus stops to be narrowly spaced, which in turn makes buses slow and unreliable; the ultimate result is that public transit competes with walking and cycling rather than cars. Given the magnitude of the problems caused by car dependency, it is necessary to provide public transit systems that can work as an alternative mode for car dependent individuals. Unfortunately, transit agencies today are suffering from budget problems, so cost-effective solutions must be found.

One well known solution to this problem is bus stop consolidation, that is, the removal of bus stops. Bus stop consolidation can lead to a variety of benefits both to passengers and to agencies: passengers will enjoy faster, more frequent, and more reliable service, while agencies will save on operating costs. The main concerns among transit agencies when discussing busstop consolidation is increased walking time and loss in demand. The first of these issues, as this paper will show, can be mitigated by ensuring that passengers who have difficulty walking are minimally affected. The second issue is a perception rather than a reality: stop consolidation has been implemented successfully in numerous cities, including Seattle and Portland, with no loss in ridership (El-Geneidy, Strathman, Kimpel, \& Crout, 2006; Kehoe, 2004). The practice of consolidation is gaining popularity among agencies, with implementations currently underway in San Francisco, Washington DC, and Toronto (SFMTA, 2010; TTC, 2014; WMATA, 2009).

The key consideration in stop consolidation is the issue of stop spacing. The ideal stop spacing strikes a balance between being wide enough to provide fast service and being narrow enough to allow passengers to walk to bus stops easily. A variety of strategies for finding this balance exist. Agencies have often taken simple approaches, either by placing stops every one or two blocks, or by using textbook spacing standards, such as a stop every 400 metres (Walker, 2012). These rule-of-thumb approaches are easy to understand, but often do not consider such factors as land use, population density, and the actual distances that passengers walk.

Conversely, the vast majority of stop-spacing research, as observed by the authors, takes an engineering approach to the subject. This approach, while mathematically rigorous, has a tendency to be very complicated and is thus difficult for agencies to apply. Additionally, the practical value of engineering approaches is often unclear, as the mathematical models typically go into great depth on minor operating details while overlooking much more important social realities of consolidation.

Thus, the goal of this paper is to develop a new methodology for bus-stop consolidation. This methodology is simple enough for agencies to understand and apply, effective enough to generate significant savings in running time and operating costs, adaptable to any city and to different agencies' specific needs, and sensitive to those who would be most affected by the removal of bus stops. In this paper we use Montreal Canada as a case study, yet the methodology developed can be applied to other transit agency. The paper starts with a review of literature on 
bus stop spacing and existing spacing methodologies; it then describes the new methodology and applies it to the entire bus network of the Société de transport de Montreal (STM), Montreal, Canada. Lastly, the savings from implementing this methodology are estimated at the system level, and directions for future research are discussed.

\section{LITERATURE REVIEW}

\subsection{Benefits and drawbacks of wider stop spacing}

There are many benefits to having wider stop spacing (El-Geneidy et al., 2006). The most obvious one, as previously mentioned, is that wider spacing allows buses to go faster (Kehoe, 2004; TTC, 2014; Walker, 2012). In addition to reducing trip times for passengers, faster buses also reduce costs for transit agencies, as fewer operating hours are required. Reduced bus running times also result in higher trip frequencies and thus, shorter waiting times; in other cases, running time reductions can be high enough to operate a route with fewer buses, representing even greater cost savings (Marchal, 2012).

There are numerous other benefits of bus stop consolidation (El-Geneidy, 2006; Gordon, 2010; Kehoe, 2004; TTC, 2014). Bus reliability can be improved, since the stops with the most inconsistent passenger activity (boarding and alighting) will be removed. Passenger comfort is improved due to less stopping and starting. Bus-stop maintenance costs decrease due to having fewer stops in the system. Traffic flow and safety improves due to buses exiting and re-entering traffic lanes less frequently. On top of these benefits, consolidation is easy to implement, given that it has no capital costs and can be tested via pilot projects.

The main drawback of wider stop spacing is increased access or egress time (i.e. walking time) for passengers (Benn, 1995; El-Geneidy et al., 2006; Furth \& Rahbee, 2000). The people who will be most affected by stop removal are those with reduced mobility; this includes people in wheelchairs, people who are injured or in poor health; people with heavy loads to carry; and people with small children. To minimize this impact, it is essential that stop removal does not occur where these populations need bus service the most.

The counter-argument to this is that passengers are willing to walk greater distances to a better transit service and that there are health benefits from doing so (Day, Loh, et al., 2014; ElGeneidy, Grimsrud, Wasfi, Tetrault, \& Surprenant-Legault, 2014; O’Sullivan and Morrall, 1996; SFMTA, 2010; Walker, 2012). Both points of view are valid, and thus, there is a delicate balance to be met between the costs and benefits of increased access and/or egress time.

\subsection{Current spacing methodologies}

As previously mentioned, several methodologies exist for determining the best stop spacing. Each has advantages, but also has serious drawbacks. The simplest method is to place stops a consistent number of blocks apart, for example, every two blocks. This method, which is used by several agencies, is straightforward, but it assumes blocks are equal length and ignores considerations of land use, population density, and the fact that people will walk greater distances to use a better service.

Another method is to use stop spacing standards such as 400 metres. This approach has 
most of the same problems cited above, and additionally, no one seems to be able to agree on a standard (BCTransit, 2010; CTA, 2001; MTA, 2000; TTC, 2014; WMATA, 2009). Some agencies bring more nuance to the problem, providing different spacing standards based on service type or built environment (BCTransit, 2010; Howe, 2011; Translink, 2012). This is an improvement over the one-size-fits-all approach, but there remains a reliance on false dichotomies such as "urban" vs. "suburban," ignoring the reality that a multitude of urban forms exist between these extremes.

Another strategy for finding the ideal stop spacing is optimization. Optimization seeks to find the minimal social cost of a bus route to the passenger and/or the transit agency. Solutions are complex, and often require advanced mathematical techniques such as dynamic programming. But despite the attempt to bring engineering rigour to the spacing problem, all the examples of optimization found in the literature are problematic. For example, Ibeas, et al. (2010) does not account for fluctuations in service frequency and allow stops to be located away from intersections, thereby increasing walking distances unnecessarily (see Giannopoulos (1989) on bus-stop placement). Mamun and Lownes (2014) make many errors in their model inputs: for example, in-vehicle time is weighted higher than access time, and estimates for both dwell time and average passenger activity are incorrect, with too-low estimations of per-passenger boarding time (1.7 seconds, versus the accepted figure of around 3 seconds (Kittelson \& Associates 2003, Dueker, Kimpel et al. 2004, Fletcher and El-Geneidy 2013)), and far-too-high estimations of perstop passenger activity (15 to 16 passengers per trip; for comparison, the morning-peak per-stop average in Montreal is 3.3). Chiraphadhanakul and Barnhart (2013) assumes that passengers will not change bus stops to walk to better service, and uses fixed dwell times at stops (an acknowledged weakness). Furth and Rahbee (2000), one of the most highly-cited optimization papers, is generally well thought out and academically defensible, but uses arbitrary weighting figures in its attempt to redistribute demand along a route.

Overall, the use of optimization has shown itself to be an interesting mathematical exercise, but ultimately, it is not the best tool for the job. The number of possible interventions in stop consolidation is very limited: a stop can either be removed or it can be moved one or two blocks along a route. Furthermore, these interventions are very crude from an engineering perspective. Unlike the problems posed by, say, bridge design or particle acceleration, the nature of the bus-stop problem is not one of millimetres and microseconds. With this in mind, it is uncertain what the advantages are of generating complex models that approximate such minutiae as the effect of traffic congestion on buses. Clearly, a simpler approach is preferable.

Lastly, all of the above methodologies have failed to properly address the sociopolitical nature of transit-service provision. Issues such as reduced passenger mobility, bus drivers' union agreements, and proximity to services like hospitals and schools are rarely discussed, if ever, despite the fact that any of these issues can instantly cripple months of painstaking calculations. Occasionally, "social costs" are considered (Ibeas, et al., 2010), but only in the context of reducing travel times or expenditures. Given the enormous political challenges of implementing stop consolidation programs (El-Geneidy, 2006), new methodologies must at very least 
acknowledge these non-technical issues if they are ever to be of value outside academia.

\section{METHODOLOGY}

\subsection{Overview}

Our methodology for deciding which stop to consolidate along a bus route consists of five main steps; see Table 1.

TABLE 1: Main steps in the new methodology, along with the factors involved in each

\begin{tabular}{l|l}
\hline Main steps & Factors considered \\
\hline 1. Determining each stop's catchment area (Section 3.2) & $\begin{array}{l}\text { Population density } \\
\text { Waiting time for bus } \\
\text { Intersection density } \\
\text { Distance to downtown }\end{array}$ \\
\hline 2. Determining each stop's class (Section 3.3) & $\begin{array}{l}\text { Needs of those with reduced mobility } \\
\text { Transit connections } \\
\end{array}$ \\
$\begin{array}{ll}\text { Passenger activity } \\
\text { First/last stops }\end{array}$ \\
\hline 3. Deciding which stops should be removed (Section 3.4) & $\begin{array}{l}\text { Catchment-area overlap (Section 3.2) } \\
\text { Bus-stop classes (Section 3.3) }\end{array}$ \\
\hline 4. Calculating the savings that result from the removal of & Current number of buses on the route \\
these stops (Section 3.5) & $\begin{array}{l}\text { Average cycle time } \\
\text { Average headway } \\
\text { Time saved per stop removed }\end{array}$ \\
\hline 5. Determining the impact on passengers (Section 3.6) & $\begin{array}{l}\text { Overall service coverage change } \\
\text { Overall travel-time change }\end{array}$ \\
\hline
\end{tabular}

These steps will now be described in detail, using bus route 161 Van Horne in Montreal as an example. This route is one of the busiest in the city, serving three subway stations and over 28,000 passengers each weekday. After demonstrating how the process works at the route level, the methodology will be automated via computer software and then applied to the entire bus network.

On a side note, it should be stated that this methodology is a preliminary step in the decision to remove stops, not the last step. The stops that this methodology recommends for removal must be assessed in-person prior to any final decisions on removal, as there are many factors at a bus stop that cannot be analyzed via software. Also the criteria used in our study can be modified based on the needs of each region to include additional criteria or exclude any of the ones we included.

\subsection{Catchment area}

Catchment areas around bus stops - that is, the area from which most of a stop's passengers originate - have traditionally thought of as fixed in size. However, El-Geneidy, Grimsrud, et al. (2014) have found that passenger walking distances to bus stops are not fixed, but variable. In 
their study, data from Montreal's origin-destination survey was used to generate a formula which approximates the $85^{\text {th }}$ percentile of passenger walking distance for each stop. This formula is:

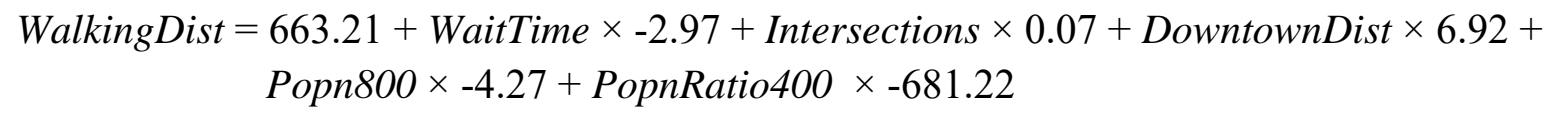

where

WalkingDist $=\quad$ measured in metres

WaitTime $=\quad$ the average wait time at the stop for the bus, specific to the route

Intersections $=\quad$ the number of street intersections within 510 metres of the stop

DowntownDist $=\quad$ the linear distance to the centre of downtown in kilometres

Popn800 = the population, in thousands, within 800 metres of the stop

PopnRatio400 $=\quad$ the proportion of the population living within 400 metres to the population within 800 metres

Geographic information systems (GIS) were used to calculate these figures for every stop in Montreal. The formula was then used to determine the size of the catchment area around each stop. These catchment areas, which were based on street-network walking distances rather than straight-line distances, ranged in radius between 255 and 832 metres, with an average of 553. For more information on the methodology behind this formula, or to learn how to estimate the parameters for a particular city, please refer to El-Geneidy, Grimsrud, et al. (2014). Agencies lacking the resources to adapt the formula can simply re-use the coefficients provided here.

\section{Catchment area \& stop spacing}

In order to balance the size of duplication and gaps in service between catchment areas (see Walker (2012)), bus stops should be spaced using the same distance as the catchment area radius. Using this principle, the edge of a stop $S$ 's catchment area will touch the stops that come before and after $S$ along the route (Figure 1). 


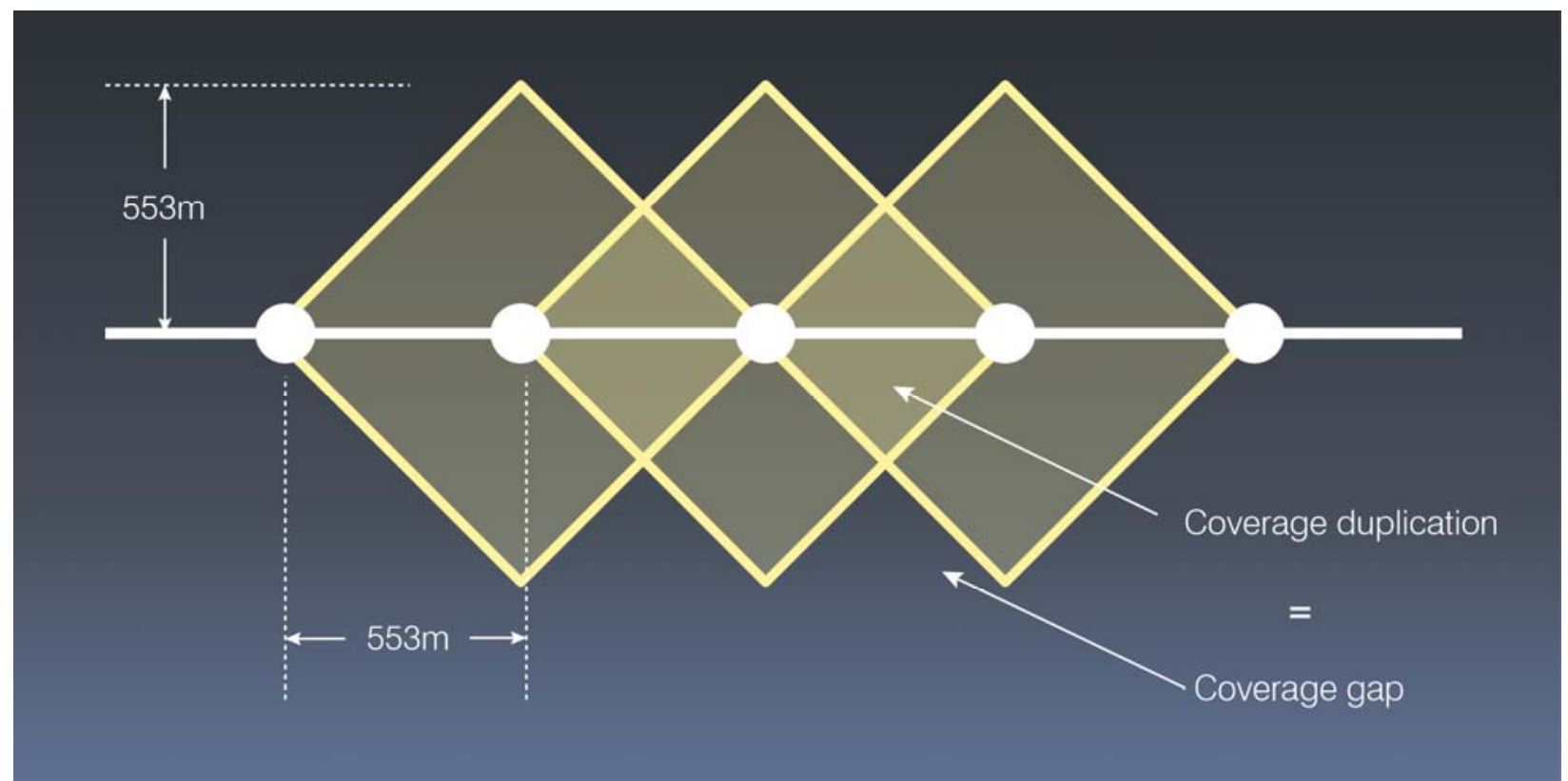

FIGURE 1: Balancing duplication and gaps in service between catchment areas

This paper loosens this principle slightly to generate the fundamental spacing rule for the new methodology: each stop $S$ should have exactly one other stop before and after it within its catchment area. If there is more than one stop on one side of $S$, then the most important stop on that side is kept, while the other stop(s) are considered for removal (Figure 2). This rule allows the balance between coverage gaps and duplications to be largely maintained while allowing more flexibility in terms of which stops to remove; on the vast majority of routes, the most important stops would not be evenly spaced.

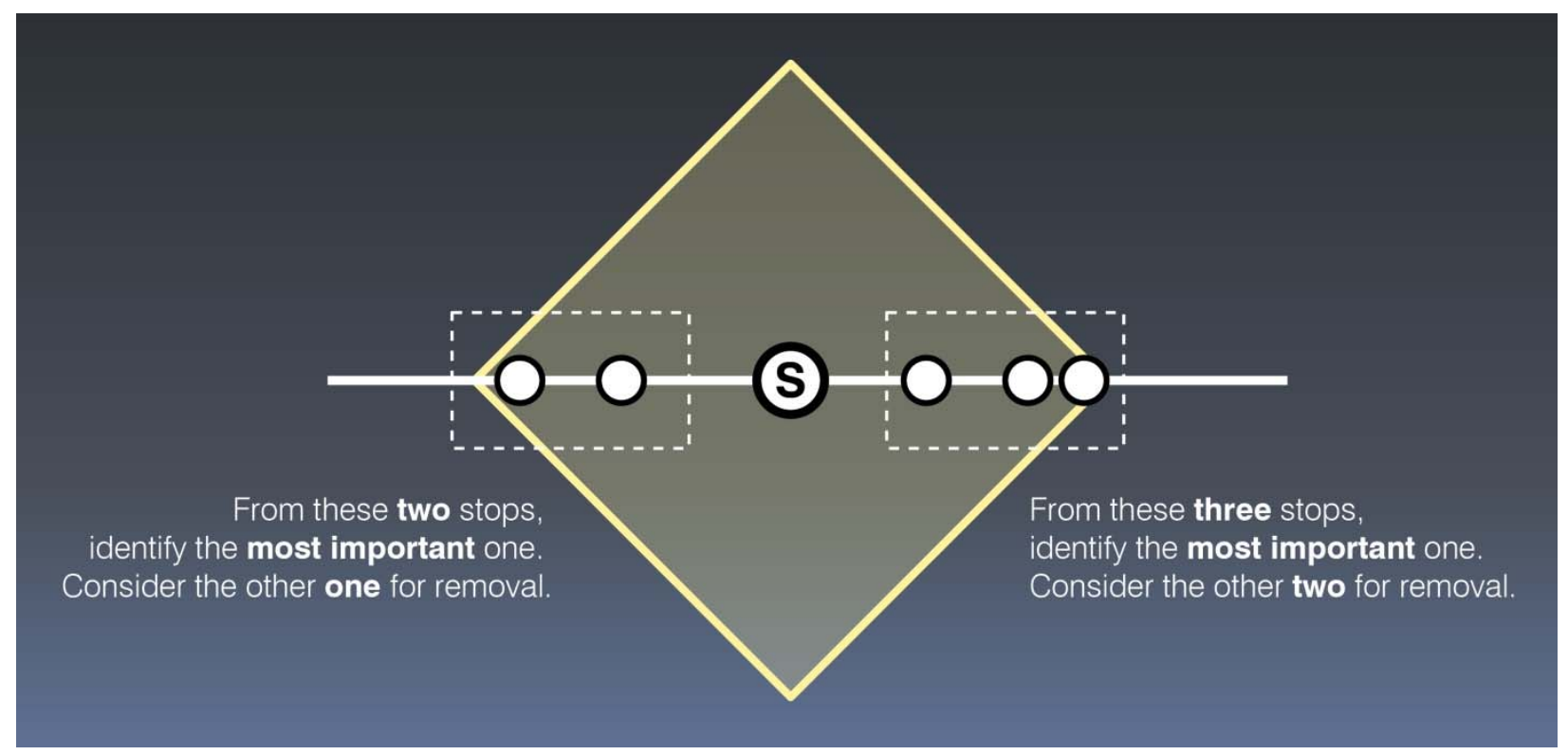

FIGURE 2: The fundamental spacing rule of the new methodology 


\subsection{Stop importance}

After catchment areas are determined, the class, or importance, of each stop is calculated. Stop importance is subjective and is based on a variety of technical and political factors; transit agencies will inevitably differ over which factors they deem important, based on their own specific needs. However, for demonstration purposes, the authors have chosen to consider the following four factors in assessing stop importance: the needs of people with reduced mobility, transit connections, passenger activity, and whether the stop is the first or last stop on the route.

Factor 1: Reduced mobility. To reduce the impact on people with reduced mobility, it was decided that stops serving high concentrations of these populations must be kept. To this end, the locations of 159 health-care centres, 300 seniors residences, and 37 hospitals on the island of Montreal were found. Stops that were considered to be "serving" these facilities were then identified. Two different methods were used here: one to identify stops serving health-care centres and seniors residences, which tend to be relatively small buildings; and one to identify stops serving hospitals, which tend to be substantially larger and have multiple entrances. Health-care centres and seniors residences were considered to be served by a stop $S$ if their midpoints were inside S's catchment area and there was no other stop on the S's route that was closer, in terms of network distance. For each hospital $H$, a more complex method was used: first, the block occupied by each hospital, and all the street intersections that acted as vertices of these blocks were identified (Figure 3.1). Second, using these vertices (intersections), the smallest possible polygon $P$ that enclosed the hospital's building footprint was drawn (Figure 3.2). Third, for each vertex $V$ composing $P$ that was inside a bus route's catchment area, the stop on this route with the closest network distance to $V$ was identified as "serving" the hospital (Figure 3.3 / 3.4). Transit agencies implementing this methodology may wish to consider additional factors to help those with reduced mobility, such as proximity to zero-car households and elderly populations.

Factor 2: Transit connections. To maintain the connectivity of the transit network, all stops that directly connect to a major transit line should be kept. For this paper, "major transit lines" were defined as being either: metros (subways), commuter trains, and buses belonging to either Montreal's ten-minute-max frequent network, its peak-only express-bus network (400-series), or its shuttle buses (700-series and shuttles for the elderly). Connections to other buses were also considered important, though less so; their role in the method will be clarified in Section 3.3.1. 
Factor 3: Passenger activity. When considering passenger activity ("pax") at each stop, volume is an important factor, but so is consistency. A stop that picks up ten passengers on one trip and zero on the next is one that will cause fluctuations in the runtime of a bus route; these fluctuations, in turn, lead to decreased reliability. A variable, pax quality was created to distinguish stops with high pax and low variation (steadily busy stops) from those with low pax and high variation (inconsistent low-demand stops). This variable was calculated as follows:

$$
\text { PaxQuality }=\frac{\text { Mean pax }}{\text { Std deviation of pax } \div \text { Mean pax }}=\frac{(\text { Mean pax })^{2}}{\text { Std deviation of pax }}
$$

The formula is based on the coefficient of variation (CV), a standarized measure of the variation of a variable, which is calculated as the standard deviation divided by the mean. However, CV only measures variation, whereas a variable that also reflects passenger volume is required; this can be achieved by dividing the mean of passenger activity by the $\mathrm{CV}$, resulting in the above formula. 

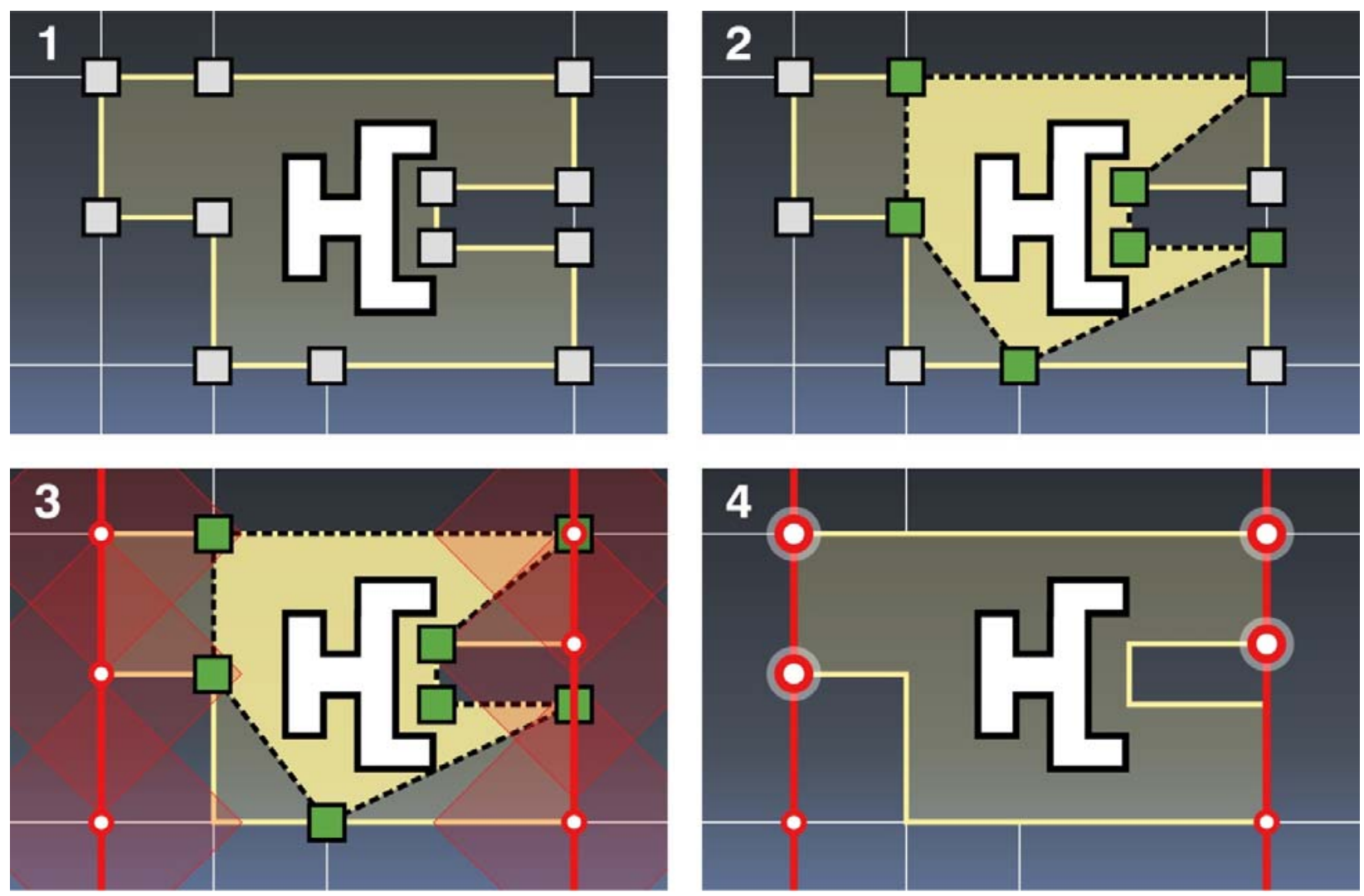

City blocks and streets

行 Building footprint for hospital $\boldsymbol{H}$

Large block $\boldsymbol{B}$ containing $\boldsymbol{H}$

Bus route and bus stop

Catchment area around bus stop
Polygon $\boldsymbol{P}$ enclosing $\boldsymbol{H}$

Street intersection / Vertex of $\boldsymbol{B}$

Street intersection / Vertex of $\boldsymbol{P}$ Used for determining which stops serve $\boldsymbol{H}$

Bus stop considered to be serving $\boldsymbol{H}$

\section{FIGURE 3: Methodology for determining the closest stops to hospitals}

(1) The building footprint of a hospital $H$, the block $B$ where it is situated, and the street intersections that form $B$.

(2) The smallest polygon $P$ enclosing the hospital building footprint using the intersections that form the block $B$.

(3) The bus lines near the hospital $H$, their stops, and the bus stop catchment areas.

(4) Stops whose catchment areas cover the intersections composing $P$; that is, the stops serving the hospital.

Factor 4: First and last stops. On many routes, the first and last stops are located for strategic reasons related to bus drivers' layovers. Thus, all first and last stops on each route are kept.

It should be noted again that there is a high degree of subjectivity in the factors which are used to determine bus-stop class. This is deliberate, since political and personal values will always play a 
strong role in the provision of transit service. Agencies are encouraged to experiment with different combinations of factors here based on their specific operating requirements, local regulations, and political values. Alternate factors for determining bus-stop importance could include proximity to schools or could account for federal discrimination laws, such as Title VI in the United States.

\section{Stop classes}

The above four factors were used to determine each bus stop's class (importance). All stops in the STM's network were categorized into six classes from A to F, with A being the most important (a "must-keep" stop) and F being the least important. The criteria for each class are as follows:

- Class A: Serves reduced-mobility facilities or Connects to the metro, train, or to major buses (frequent / express / shuttle) or Is a first or last stop

- Class B: Fourth (top) quartile of pax quality

- Class C: Connects to regular bus network

- Class D: Third quartile of pax quality

- Class E: Second quartile of pax quality

- Class F: All other stops

The classes for each stop on Route 161 and their determining factors are shown in FIGURE 4. As with class factors, agencies are encouraged here to experiment with different classifications. Future variations of this methodologies could use continuous values for importance instead of classes. 


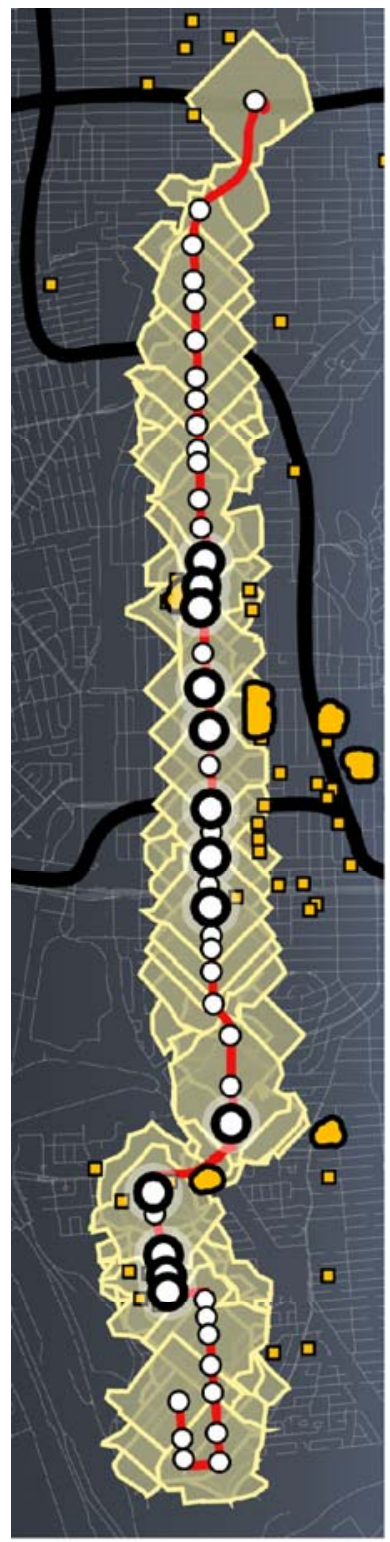

Reduced mobility

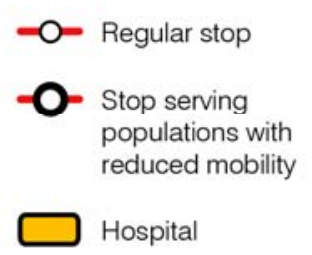

a Health-care or seniors' centre

Catchment area

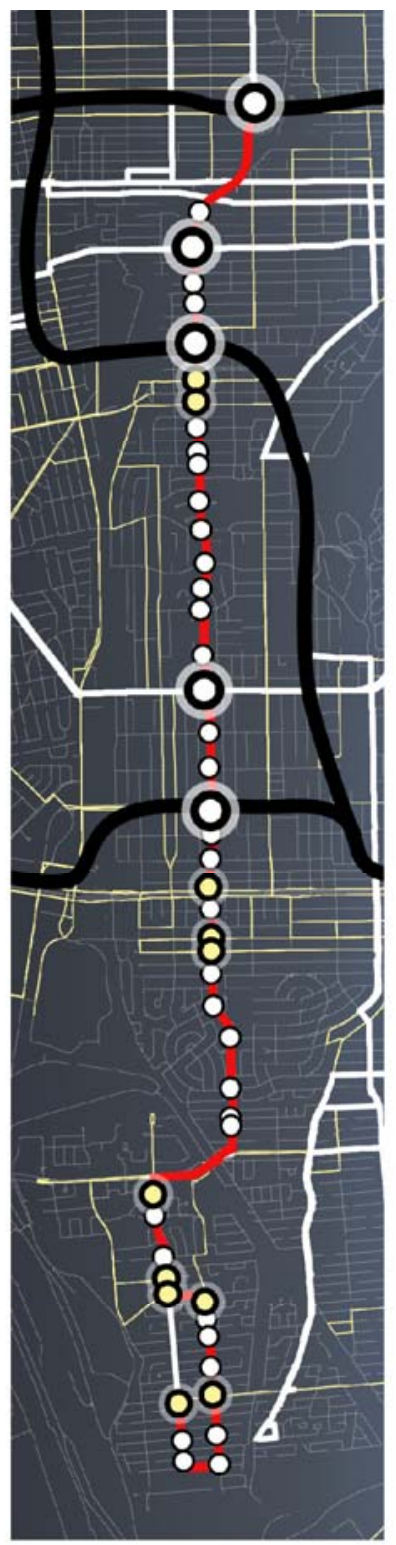

\section{Public transit}

- - Connection to minor transit - Connection to
major transit

= Minor buses

$=$ Major buses

$\longrightarrow$ Métro

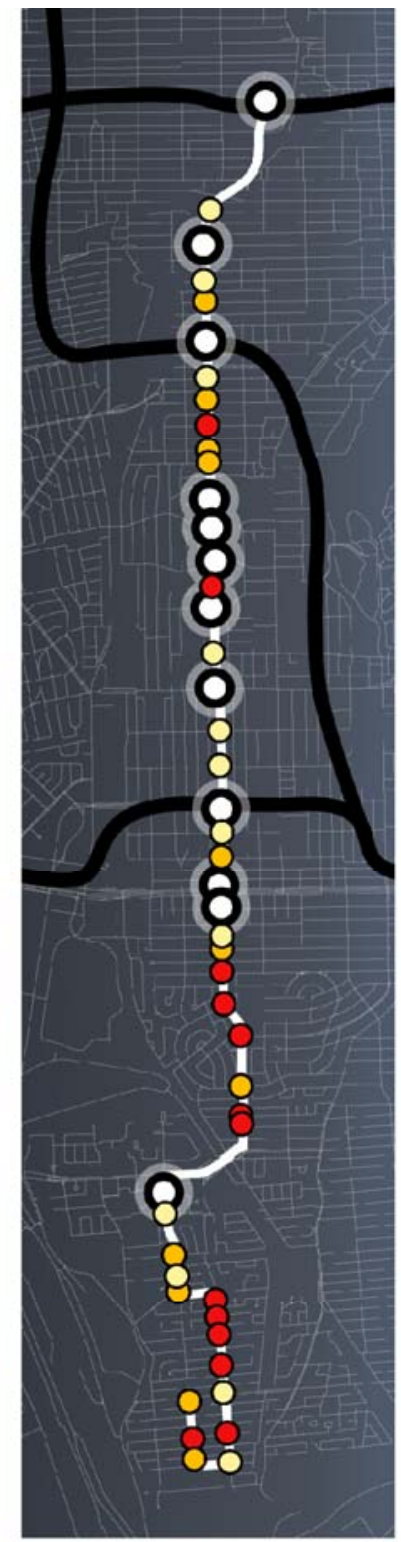

Pax quality

( Quartile 4

O Quartile 3

○ Quartile 2

- Quartile 1

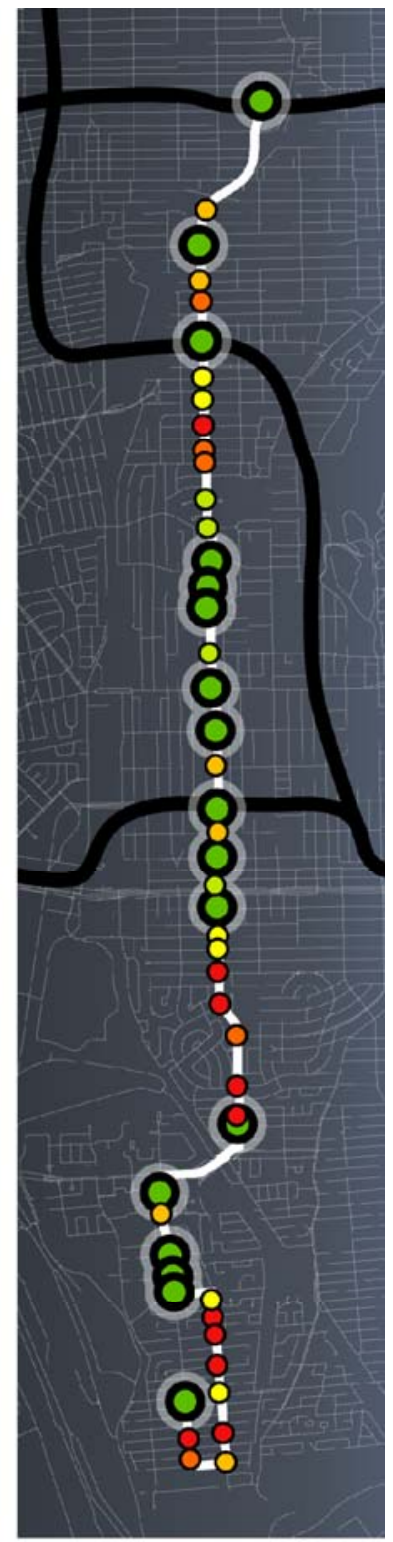

Classes

- Class A

O Class $\mathbf{B}$

O Class $\mathbf{C}$

O Class D

O Class E

- Class $\mathbf{F}$ 


\subsection{Selecting stops for removal}

Removal score

Catchment areas and classes are the two main factors used to determine the removal score of each stop. Stops with a removal score greater than zero will be considered for removal, and stops with higher scores will have a greater chance of being removed. Two other factors come into the final decision of whether to remove a stop: twin stops and consecutive stops. Twin stops are stops on a route that serve the same intersection, but in the opposite direction. For consistency, either both stops in a twin pair will be kept or both will be removed. More specifically, if either stop in a pair of twins has a removal score of zero, both will be kept. Consecutive stops will not be removed, so as to avoid creating excessively wide spacing. If there are consecutive stops that all have removal scores greater than zero, only the odd- or even-numbered stops will be removed, depending on which have the higher average removal score. Ties between removal score averages can be broken using the average pax quality.

\section{The process}

One of the aims of this research was to generate a systematic process of selecting stops for removal so that it could be automated via computer software for an entire bus network. This process is as follows:

1. Give each bus stop in the system an initial removal score of 0 .

2. For each route $R$ in the system, for each direction $D$ in $R$, and for each stop $S$ along $D$ :

a. Find the stops on route $R$ in direction $D$ that fall within $S$ 's catchment area.

b. Find the most important stop before and after $S$ within the catchment. Importance is determined first by class and second by pax quality. See Figure 5.

c. If there are other stops within S's catchment, and if they are of lower importance than $S$, and if they are not Class A stops, add one point to their removal scores.

3. For each stop $S$ with a removal score of at least 1:

a. If $S$ has a twin stop, and the twin of $S$ has a removal score greater than zero, mark $S$ and its twin as under consideration

b. If $S$ has no twin stop, mark it as under consideration

4. For each stop $S$ under consideration that is not beside other stops under consideration:

a. Remove $S$

5. For all the groups of consecutive stops on a route that are under consideration:

a. Calculate the average removal score of the odd- and even-numbered stops

b. If the odd-numbered stops have a higher average removal score, remove them, and vice versa. Break ties on average pax quality. 


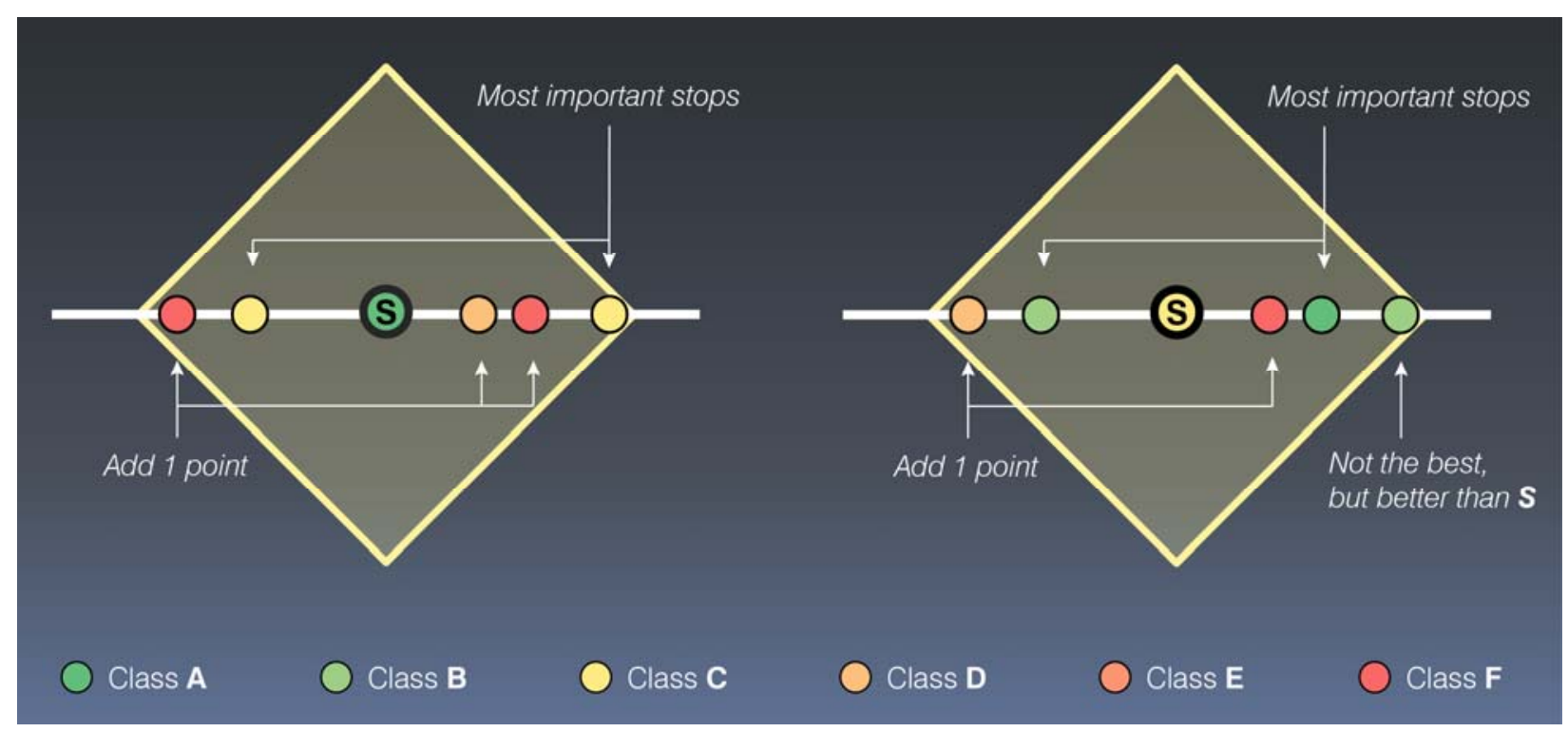

FIGURE 5: The process for calculating the removal score of stops, based on catchment areas and stop classes

Table 2 gives an example of the scoring process for part of Route 161. The first stop, Station Rosemont, has no stops before or after it within its catchment; as such, there are no stops to consider removing, and no points to be given. For the second stop, there is only one stop after it, so there is no need to remove any stops or give any points.

For stop 3, there are two stops after it within its catchment; of these, stop 4 is the most important, based on its class, so the other stop, number 5, is given a point. For stop 4, there is only one other stop before and after it within its catchment, so no points are given. For stop 5, there are two stops before it within its catchment; however, both of these stops have greater importance than stop 5, so no points are given. For stop 6, there are two stops after it; these two stops have equal classes (C), but stop 8 has a lower pax quality and is thus given a point.

TABLE 2: Example of removal score calculation on Route 161

\begin{tabular}{l|cc|cc|c}
\hline Stop name & Class & PaxQuality & Stops before & Stops after & Score \\
\hline 1. Station Rosemont & $\mathrm{A}$ & .98 & 0 & 0 & - \\
2. Van Horne / Waverly & $\mathrm{D}$ & .63 & 0 & 1 & - \\
3. Van Horne / du Parc & $\mathrm{A}$ & .83 & 1 & 2 & - \\
4. Van Horne / Querbes & $\mathrm{D}$ & .52 & 1 & 1 & - \\
5. Van Horne / Bloomfield & $\mathrm{E}$ & .50 & 2 & 1 & $\mathbf{1}$ \\
6. Station Outremont & $\mathrm{A}$ & .92 & 1 & 2 & - \\
7. Van Horne / McEachran & $\mathrm{C}$ & .73 & 1 & 2 & - \\
8. Van Horne / Rockland & $\mathrm{C}$ & .46 & 2 & 2 & $\mathbf{1}$ \\
9. Van Horne / Hartland & $\mathrm{F}$ & .10 & 2 & 2 & $\mathbf{4}$ \\
10. Van Horne / Pratt & $\mathrm{E}$ & .31 & 2 & 2 & $\mathbf{1}$ \\
11. Van Horne / de Vimy & $\mathrm{E}$ & .42 & 2 & 1 & - \\
\hline
\end{tabular}


After this process is completed for each stop in both directions of Route 161, twin stops must be considered. Table 3 shows a selection of stops from Route 161, along with their removal scores in both directions. For the first stop in the table, stop 39, the westbound direction has a score greater than zero, and there is no twin; as such, it can be considered for removal.

For stops 40 and 41, both their westbound and eastbound twins have scores greater than zero, so both can be removed. In the case of stop 42 though, the eastbound stop has a score greater than zero, but its westbound twin does not; as such, the pair is not considered for removal.

TABLE 3: Example of how to deal with twin stops on Route 161

\begin{tabular}{|c|c|c|c|}
\hline Stop name & $\begin{array}{c}\text { WESTBOUND } \\
\text { Score }\end{array}$ & $\begin{array}{c}\text { EASTBOUND } \\
\text { Score }\end{array}$ & Consider removing \\
\hline 39. Guelph / Parkhaven & 1 & (no twin) & Yes \\
\hline 40. Guelph / Whitehorne & 5 & 5 & Yes \\
\hline 41. Guelph / Melling & 3 & 1 & Yes \\
\hline 42. Guelph / McMurray & - & 2 & No \\
\hline 43. Guelph / Westminster & - & - & No \\
\hline 44. Guelph / Westluke & 3 & (no twin) & Yes \\
\hline
\end{tabular}

Lastly, consecutive stops must be considered. Table 4 shows all the groups of consecutive stops under consideration for removal. For the first pair, stops 9 and 10, the average removal score of the odd and even stops is calculated; the average for stop $9((4+4) \div 2=4)$ is higher than that of $10((1+3) \div 2=2)$, so it is removed.

For the second group of consecutives, stops 12 and 13, all the removal scores are the same (1), so the average pax qualities are calculated. Stop 12 has the lower average pax quality, so it is removed.

For the third group, the average removal score for the odd stops (stops 27 and 29) is 2.5, which is higher than the average for the even stops, so the odd stops are removed. The reverse situation occurs in the last group, where the even-numbered stop in the middle (stop 40) has a higher average score than that of the odd-numbered stops, so only it is removed. 
TABLE 4: How to deal with consecutive stops under consideration for removal

\begin{tabular}{l|c|cc|cc|c}
\hline & & \multicolumn{2}{|c|}{ WESTBOUND } & \multicolumn{2}{c|}{ EASTBOUND } & \\
Stop name & $\begin{array}{c}\text { Consider } \\
\text { removing }\end{array}$ & Score & PaxQual & Score & PaxQual & Remove \\
\hline 9. Van Horne / Hartland & Yes & 4 & 0.10 & 4 & 0.05 & Yes \\
10. Van Horne / Pratt & Yes & 1 & 0.31 & 3 & 0.23 & No \\
\hline 12. Van Horne / Northcrest & Yes & 1 & 0.85 & 1 & 0.84 & Yes \\
13. Van Horne / Wilderton & Yes & 1 & 0.90 & 1 & 0.89 & No \\
\hline 27. Van Horne / Macdonald & Yes & 2 & 0.29 & 1 & 0.16 & Yes \\
28. Fleet / Finchley & Yes & 1 & 0.19 & 1 & 0.14 & No \\
29. Fleet / Netherwood & Yes & 3 & 0.13 & 4 & 0.02 & Yes \\
\hline 39. Guelph / Parkhaven & Yes & 1 & 0.25 & & (no twin) & No \\
40. Guelph / Whitehorne & Yes & 5 & 0.02 & 5 & 0.09 & Yes \\
41. Guelph / Melling & Yes & 3 & 0.17 & 1 & 0.27 & No \\
\hline
\end{tabular}

\subsection{Savings calculations}

At this point, runtime savings from stop removal can be calculated. For this research, savings are calculated for the morning peak period (6:30 to 9:30), using General Transit Feed Specification (GTFS) schedule data. This period was used for analysis as it tends to have the most frequent, and therefore most costly, bus service of the day (Walker, 2012); it is thus the ideal place to look for savings. Since there is considerable variation in service level and congestion during this interval, savings were calculated separately for half-hour periods (6:30 to 6:59, 7:00 to 7:29, etc.). The following process is used:

First, the current number of buses on the route, that is, those either actively serving the route or on layover, is determined. Second, the average cycle time for the route is calculated; this consists of the bus's total runtime in both directions plus layover time. Third, the average headway is calculated by dividing the average cycle time by the current number of buses. Fourth, the time savings expected from removing the selected stops were determined. The average time saved by removing one stop is about 12 seconds (Stewart \& El-Geneidy, 2014; Tétreault \& ElGeneidy, 2010); this comprises time spent accelerating, decelerating, and operating the bus doors. However, if a stop is not used on each trip, the savings will be lower; for example, if a stop $S$ is only used on every other trip, the average savings from removing $S$ will be 6 seconds instead of 12. In general, if a stop $S$ has an average pax per trip of at least 1, the savings from removing $S$ are 12 seconds; otherwise, the savings are calculated as $S$ 's average pax per trip multiplied by 12 seconds. Fifth, the new cycle time is calculated by subtracting the total time savings from the cycle time. Sixth, the new headway is determined by dividing the new cycle time by the current number of buses. Seventh, the new number of buses required to maintain the existing headway is calculated by dividing the new cycle time by the existing headway. If the new number of required buses, (rounded up) is less than the existing number of required buses (rounded up), then it is possible to operate the route at existing frequencies with fewer buses.

The possibility of removing a bus was explored further, since it represents such 
substantial savings. Specifically, the increase in headway was calculated for a hypothetical situation where one less bus was used compared to the current number; for example, if 17 buses are currently being used on a route, the headway with only 16 buses would be calculated, after taking the runtime savings from stop removal into account. In doing this, it was found that numerous routes could be run with one less bus with very minor increases in headway.

The question that gets raised here is how much of an increase in headway is acceptable in order to obtain the considerable savings of needing one less bus on a route. Agencies will differ in how much they are willing to allow headways to increase, but for illustrative purposes, a 5\% increase will be used. To put this figure in context, a 5\% headway increase on a route that has a ten-minute headway would amount to just an extra 30 seconds between buses. However, it is also necessary that this increase of $5 \%$ or less occurs over a long enough period of time for a bus to complete one full trip cycle. For example, if a route $\mathrm{X}$ with a cycle of 75 minutes saw a headway increase of just 3\% in one half-hour period but an increase of $20 \%$ in the previous and subsequent half-hour periods, then clearly a bus cannot be saved (Table 5); this route would need minor headway increases over at least 75 minutes, which is to say, at least three consecutive halfhour periods. However, if a route $\mathrm{Y}$ with a cycle of 105 minutes had headway increases of 5 or less in at least four consecutive half-hour periods (120 minutes), then this is considered acceptable for this paper (see Table 5).

TABLE 5: Increase in headway with one less bus on hypothetical routes. Routes $Y$ and $Z$ can operate using one less bus with only minimal increases in headway, whereas route $X$ cannot.

\begin{tabular}{c|cc|cccccc}
\hline Route & $\begin{array}{c}\text { Average } \\
\text { cycle } \\
\text { time }\end{array}$ & $\begin{array}{c}\text { Half- } \\
\text { hour } \\
\text { periods } \\
\text { needed }\end{array}$ & \multicolumn{6}{|c|}{ Increase in headway with one less bus } \\
\hline $\mathbf{X}$ & 75 & 3 & $32 \%$ & $20 \%$ & $\mathbf{3 \%}$ & $20 \%$ & $14 \%$ & $18 \%$ \\
$\mathbf{Y}$ & 105 & 4 & $19 \%$ & $\mathbf{4 \%}$ & $\mathbf{- 2 \%}$ & $\mathbf{2 \%}$ & $\mathbf{0 \%}$ & $14 \%$ \\
$\mathbf{Z}$ & 45 & 2 & $13 \%$ & $21 \%$ & $15 \%$ & $11 \%$ & $\mathbf{- 1 \%}$ & $\mathbf{4 \%}$ \\
\hline
\end{tabular}

\subsection{Impact on passengers}

Lastly, the impact on passengers that results from removing stops was determined. This impact was measured in two ways: by decline in service coverage area, and by change in overall travel time. Coverage area decline was measured by comparing the total area covered by the route's catchment areas before and after removing stops. Change in overall travel time was calculated by finding the average change in walking time, waiting time, and in-vehicle time. 


\subsection{Comparisons with other methodologies}

For comparison, two other stop-removal methodologies were applied to the route 161. The first of these was the 400-metre "rule of thumb." The exact method of applying this rule to a bus route is open to interpretation. For this paper, the following approach was used:

1. Calculate the distance, Dist, between the first and second stops, $A$ and $B$

2. If Dist $>400$, keep both $A$ and $B$, then repeat step 1 with the next consecutive pair of stops

3. Otherwise:

a. Repeat the following process until Dist $>400$ :

i. Calculate the difference, Diff1, between 400 and Dist

ii. Add to Dist the distance to the next stop, $X$

b. Calculate the difference, Diff2, between 400 and the new value of Dist

c. If Diff $1<$ Diff2, remove all the stops from $B$ up to two stops before $X$

d. Otherwise, remove all the stops from $B$ up to one stop before $X$

e. Repeat from step 1, with the next consecutive pair of remaining stops

See Table 6 for an example of this process.

TABLE 6: Example of how the 400-metre spacing standard was applied

\begin{tabular}{cccc}
\hline Stop & Distance from previous & Result & New distance from previous \\
\hline 1 & 0 & Keep & 0 \\
2 & 500 & Keep & 500 \\
3 & 200 & REMOVE & - \\
4 & 100 & Keep & 300 \\
5 & 350 & Keep & 350 \\
6 & 200 & REMOVE & - \\
7 & 100 & REMOVE & - \\
8 & 150 & Keep & 450 \\
\hline
\end{tabular}

The second methodology applied was an optimization approach described by Wagner and Bertini (2014). This method, like many optimization approaches, aims to minimize costs and maximize benefits; in this case, the unit of measurement is the sum of walking time and in-vehicle time incurred by all passengers on a route. A benefit-cost ratio (BCR) of removing each stop is calculated, and stops with a BCR of greater than 1 are removed. The principle variables used in determining the BCR are stop spacing and passenger activity.

The results of applying both methodologies to Route 161, which has 92 stops over both directions, is shown in Table 7. The 400-metre rule, which only considers stop spacing, is blind in its choice of stops to cut: numerous stops serving hospitals, health-care centres, and major transit connections are cut, and on many occasions, only one stop in a set of twin stops is removed. The optimization process is smarter, in that it favours stops with high ridership; however, this means that only the major transit connections are spared, while everything else is 
fair for removal. Ultimately, nearly a third of the stops selected by the 400-metre rule are problematic in some way, as are over half of those selected by the optimization method. By comparison, the methodology described in this paper has none of these problems.

TABLE 7: Comparison of different stop-removal methodologies applied to Route 161

\begin{tabular}{l|c|ccc|c|c}
\hline Methodology & Stops cut & Hospitals & Health care & Major transit & Twins separated & Problematic \\
\hline This paper's & $20(22 \%)$ & 0 & 0 & 0 & 0 & $0 \%$ \\
400m spacing & $39(42 \%)$ & 2 & 6 & 4 & 3 & $12(31 \%)$ \\
Optimization & $25(27 \%)$ & 3 & 5 & 0 & 6 & $14(56 \%)$ \\
\hline
\end{tabular}

\subsection{Application of the methodology to the entire system}

The methodology, which until now has been described at the route level, was automated using Python programming and applied to all bus stops and routes operated by STM in Montreal during the morning peak. GTFS data from 2012 was used. In this data set, there are 177 bus routes serving 8628 physical stops. There were 15,832 "logical" stops, that is, those serving different routes, directions, or trajectories (the specific sequence of stops taken by a bus). Of these logical stops, $3443(22 \%)$ serve reduced-mobility facilities and 7032 (44\%) connect to transit. Overall, 6878 (43\%) of stops were categorized as Class A (must-keep). The average stop catchment area was 553 metres.

\section{SYSTEM LEVEL ANALYSIS}

The main results from the system-level analysis are shown in Table 8 . The average increase in stop spacing is 74 metres; this means that the average increase in walking distance is just 37 metres. As well, the decline in service area at the system level is only $1 \%$.

The major benefits from the consolidation come in the form of saved buses. The number of buses that can be removed from a route while maintaining similar headways depends on how much of a headway increase an agency is willing to tolerate. Following the method described in Table 5 (Section 3.5), it is found that between 8 and 75 buses are able to be saved in the AM peak, with a maximum allowed headway increase ranging from $0 \%$ to $10 \%$. See Table 8 . Given the potential cost savings of the consolidation, particularly from the removal of this number of buses, the minor impacts on walking distance and service coverage seem like a reasonable tradeoff. 
TABLE 8: Summary of main system-level findings

\begin{tabular}{|c|c|}
\hline \multicolumn{2}{|l|}{ Number of stops removed } \\
\hline Average for each route & $14(19 \%)$ \\
\hline System-wide & $1977(23 \%)$ \\
\hline \multicolumn{2}{|l|}{ Stop spacing } \\
\hline Average increase & 72 metres $(26 \%)$ \\
\hline Average new spacing & 350 metres \\
\hline \multicolumn{2}{|l|}{ Service area } \\
\hline Average decrease for each route & $2.6 \%$ \\
\hline System-wide decrease & $1.06 \%$ \\
\hline \multicolumn{2}{|l|}{ Runtime savings (AM peak) } \\
\hline Average decrease per route & 1.2 minutes \\
\hline Total operating time saved & 109 hours ( 4.5 days) \\
\hline Average decrease in headway & 19 seconds \\
\hline \multicolumn{2}{|l|}{ Bus removal (AM peak) } \\
\hline Routes from which a bus can be removed ( $0 \%$ headway increase) & 8 \\
\hline Routes from which a bus can be removed ( $5 \%$ headway increase) & 48 \\
\hline Routes from which a bus can be removed ( $10 \%$ headway increase) & 75 \\
\hline
\end{tabular}

Different results were observed between regular, express, and frequent bus services. Generally, the express routes saw the fewest number of removed stops, since they typically have wider spacing than the regular and frequent routes. As such, the express routes also have the lowest increases in stop spacing and the lowest decreases in service area. As for decreases in runtime and headway, the frequent routes perform the best. This is expected: high frequency routes typically have consistent passenger activity at every stop, which means that the full 12 seconds of potential savings will be obtained with a higher proportion of stops removed. See Table 9. 
TABLE 9: Consolidation results by bus service type

\begin{tabular}{l|c|cccc}
\hline \multirow{2}{*}{ Stops cut } & & \multicolumn{4}{|c}{ ROUTE TYPE } \\
& & Regular & Express & Frequent & ALL \\
\hline \multirow{2}{*}{ Increase in stop spacing } & Number & 15.4 & 6.2 & 16.9 & 13.9 \\
& Percentage & $20.8 \%$ & $10.9 \%$ & $20.4 \%$ & $18.9 \%$ \\
\hline \multirow{2}{*}{ Decrease in service area } & Metres & 77.0 & 74.4 & 67.5 & 73.9 \\
& Percentage & $27.1 \%$ & $11.7 \%$ & $27.0 \%$ & $24.4 \%$ \\
\hline Decrease in runtime & Square Kilometres & 0.20 & 0.11 & 0.23 & 0.19 \\
& Percentage & $2.8 \%$ & $1.3 \%$ & $2.9 \%$ & $2.6 \%$ \\
\hline Decrease in headway & Minutes & 1.2 & 0.5 & 2.2 & 1.2 \\
& Percentage & $1.8 \%$ & $0.7 \%$ & $\mathbf{2 . 9 \%}$ & $1.8 \%$ \\
\hline
\end{tabular}

Another identified benefit from the consolidation was the reduction in service-coverage redundancy. Figure 6 shows the number of catchment areas overlapping each 100-by-100 metre grid cell on the island of Montreal, before and after removing stops. Some redundancy is useful in a transit system, but after a point, it becomes wasteful. By removing the stops identified in this study, the number of heavily overlapped areas will decline considerably.

\section{Change in travel time}

Table 10 gives a rough approximation of changes in overall passenger trip times at the system level. After considering changes to walking, waiting, and in-vehicle time, it was found that, on average, there is virtually no difference in overall trip times. It can thus be said, that passengers, on average, will see negligible differences, let alone negative impacts, on their trip times. As mentioned previously though, it will be necessary to perform more refined analysis using data from the origin-destination survey to accurately measure travel-time changes; this will allow impacts to individuals to be better quantified, and to determine how many people will be affected, particularly those with mobility impairments. 

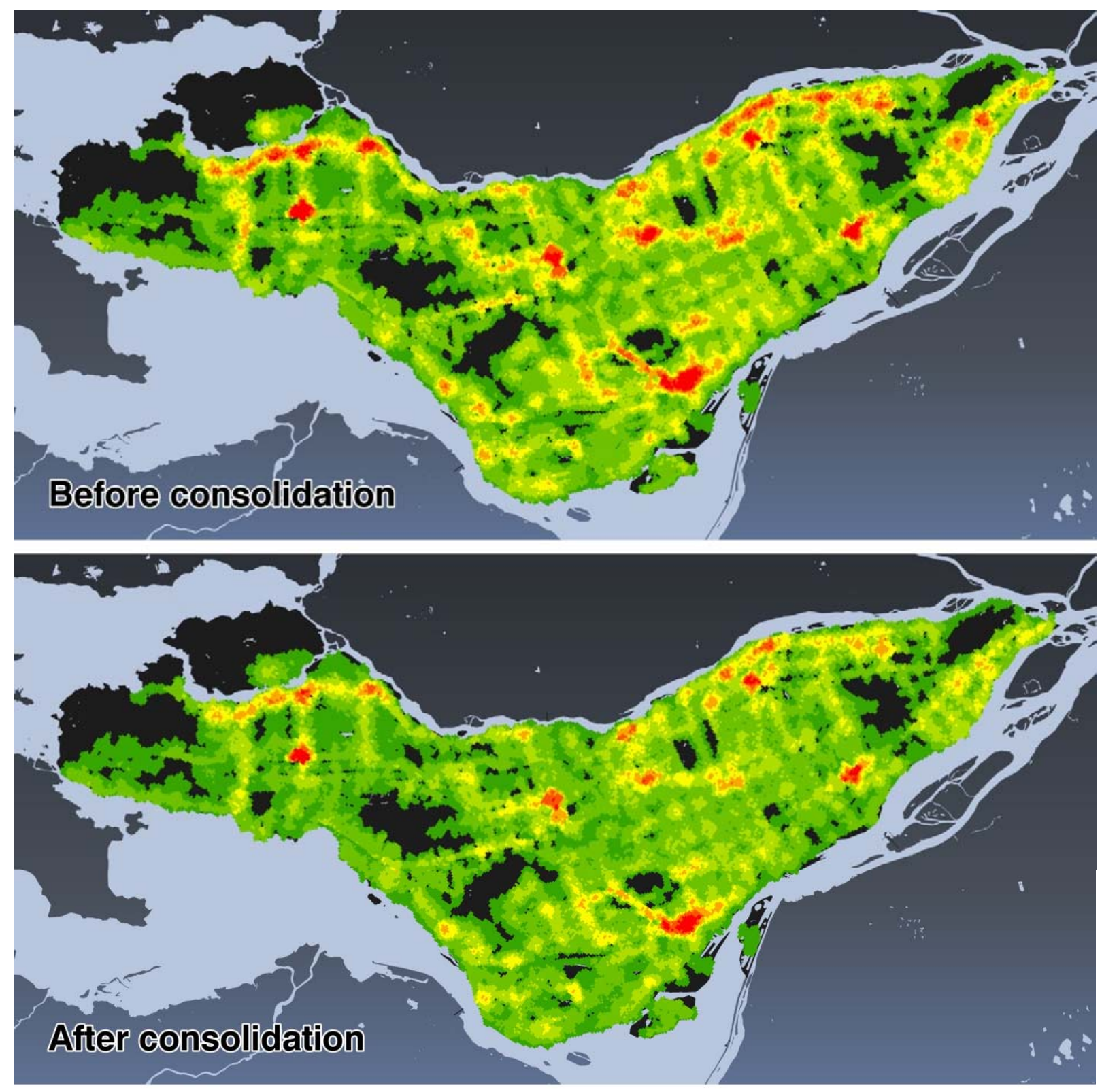

Number of overlapping bus-stop catchment areas
0: No service
$40-54$
$1-9$
$55-69$
$10-24$
$70-84$
$25-39$
$85-141$

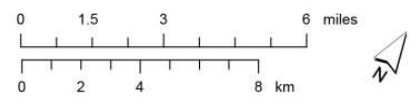

Data source: STM GTFS

Projection: NAD 1983 MTM

FIGURE 6: Reduction in service-coverage redundancy 
TABLE 10: Average change in passenger travel time at the system level

\begin{tabular}{|c|c|c|}
\hline & Change & Notes \\
\hline \multicolumn{3}{|l|}{ Walking time } \\
\hline Average increase in stop spacing & 72 metres & \\
\hline $\begin{array}{l}\text { Average increase in walking } \\
\text { distance }\end{array}$ & 36 metres & Half the stop spacing increase \\
\hline Average walking-time increase & 26 seconds & Average walking speed of $5 \mathrm{~km} / \mathrm{h}^{*}$ \\
\hline \multicolumn{3}{|l|}{ Waiting time } \\
\hline $\begin{array}{l}\text { Average AM-peak headway } \\
\text { decrease }\end{array}$ & 19 seconds & \\
\hline Average wait-time savings & 10 seconds & Half the headway decrease \\
\hline \multicolumn{3}{|l|}{ In-vehicle time } \\
\hline Average AM-peak runtime savings & 36 seconds & \\
\hline Average in-vehicle time savings & 18 seconds & $\begin{array}{l}\text { Half the runtime savings, assuming the average } \\
\text { passenger is riding half the total route }\end{array}$ \\
\hline \multicolumn{3}{|l|}{ Total change, no buses removed } \\
\hline Total change in trip travel time & -2 seconds & $\begin{array}{l}\text { Walking-time increase minus in-vehicle } \\
\text { savings minus wait-time savings }\end{array}$ \\
\hline
\end{tabular}

*Browning, et al. (2006) 


\section{CONCLUSIONS}

For this paper, a new methodology for bus-stop consolidation was developed. The overall goal was to overcome the drawbacks of current methodologies; that is, to create a process that was simple, effective, socially responsible, and adaptable not only to the needs of different transit agencies but also to any bus route.

The proposed methodology has five broad steps that can be applied to any bus route in any system: first, each stop's catchment area is found; second, the class of each stop is calculated; third, the catchments and classes are used to determine which stops should be removed; fourth, the runtime savings are calculated; and fifth, the impact on passengers is assessed. Transit agencies can apply different weights in the class calculations to set their priorities. In the current case we placed walking distances and being near a vulnerable group (hospitals or senior center) as part of the criteria.

The methodology was first tested on one bus route in Montreal, the 161, and then was automated using Python scripts for all routes operated by La Société de transport de Montréal (STM). Nearly 2000 stops were identified as candidates for removal, representing almost a quarter of all stops in the system. The runtime savings that would result from removing these stops could save as much as 109 hours of operating time during the morning peaks, and as many as 75 bus routes could operate at existing frequencies with one less bus. The routes that benefit the most from bus-stop consolidation tend to be those with the shortest headways, as they require lower running-time savings in order to save a bus.

The ultimate question for any stop-consolidation scheme is whether the operating cost savings will come at the expense of passengers. This study concludes that passengers will not be inconvenienced in any significant fashion. On average, walking times will increase by a mere 26 seconds, the entirety of which will be offset by decreases in waiting and in-vehicle time. Additionally, the overall decline in system coverage is just 1\%. The impact of the proposed stop removal on passengers is thus small.

\subsection{Future research}

There are several directions for future research leading from the results of this paper. The first is a more detailed analysis of the consolidation's effect on passengers - particularly those with mobility impairments - using data from the origin-destination survey. Given that stop removal can be met with considerable resistance from citizens, it is of utmost importance to understand what the real impact of widespread stop removal will be.

Second, for the routes that stand to benefit the most from consolidation, more detailed studies should be undertaken. The methodology presented here is only a first step to understanding potential benefits of stop-removal. Automated vehicle location (AVL) data should be used to provide more accurate estimates of savings, and on-the-ground studies must be carried out to see what considerations have been overlooked by this methodology. Lastly, pilot consolidation projects can be run on one or more routes. Such pilot projects would have very low costs, as the only major requirement would be to temporarily close selected stops. 
Removing stops from a system can be costly on the transit agencies, the approach explained in this paper can be tested by placing a temporary closed sign at suggested stops. If the savings are confirmed, then the removal can take place. Another approach is to remove two consecutive stops and replace them with a new one in the middle, yet in reality the costs associated to this approach can be much higher even at the testing level. Such approach can be implemented as a second step after implementing the suggested methodology in this paper as it will require a higher level of intervention from the transit agencies. A third direction of future research, after selecting and consolidating the suggested stops based on the methodology described in here, is to rerun the methodology again looking for two consecutive stops that score highly on the removal scale and has an intersection between them where a new bus stop can be added. Doing so will require a re-evaluation of the time savings and comparing it to the costs of removing two stops and adding a new one. Also the catchment area around the new stop has to be evaluated to ensure that such change will not be harming existing users.

\subsection{Research implications}

An overarching concern that this paper attempts to address is the disconnect between academic research and industry practice in transportation. Given the applied nature of the field, the authors believe that no transportation research is useful if it has no direct application to policy or practice. The authors have been critical of previous research on bus-stop consolidation for this reason; too much of it has approached the consolidation problem from an unnecessarily detailed scale, or has ignored the sociopolitical aspects of transit planning that can render the most meticulous calculations irrelevant. To the authors' knowledge, this paper is the first on bus-stop consolidation that considers both the technical and sociopolitical aspects of transit provision. The paper offers a methodology that can be easily adopted by transit agencies. It is thus hoped that this paper encourages researchers to take a holistic view on transportation problems, and in doing so, to help close transportation's academic/industry gap.

\section{ACKNOWLEDGEMENTS}

The authors wish to thank Michel Tremblay, Anna Guinzbourg, Sébastien Gagné at STM for providing the data to test our methodology, and providing feedback on this research. STM has provided the data used in this research for educational purpose and to help the authors in testing the methodology, STM is not responsible of any of the findings as they represents the authors ideas and efforts using Montreal as a case study. Many thanks to the members of Transportation Research at McGill (TRAM) for their support and feedback at different stages of this study. Last but not least we would like to thank the anonymous reviewers for their feedback in the rounds of review that greatly strengthened the manuscript.

\section{REFERENCES}

BCTransit. (2010). Infrastructure Design Guidelines.

Benn, H. (1995). Synthesis of transit practice 10: Bus route evaluation standards: Transportation Research Board. 
Browning, R. C., Baker, E. A., Herron, J. A., \& Kram, R. (2006). Effects of obesity and sex on the energetic cost and preferred speed of walking. Journal of Applied Physiology, 100(2), 390398.

Chiraphadhanakul, V., \& Barnhart, C. (2013). Incremental bus service design: Combining limited-stop and local bus services. Public Transport, 5, 53-78.

CTA. (2001). Chicago Transit Authority Service Standards.

El-Geneidy, A. (2006). The use of advanced information technology in urban public transportation systems: An evaluation of bus stop consolidation. (Doctor of Philosophy Dissertation), Portland State University.

El-Geneidy, A., Grimsrud, M., Wasfi, R., Tétrault, P., \& Surprenant-Legault, J. (2014). New evidence on walking distances to transit stops: identifying redundancies and gaps using variable service areas. Transportation, 41, 193-210.

El-Geneidy, A., Strathman, J., Kimpel, T., \& Crout, D. (2006). The effects of bus stop consolidation on passenger activity and transit operations. Transportation Research Record, 1971, 32-41.

Furth, P. G., \& Rahbee, A. B. (2000). Optimal bus stop spacing through dynamic programming and geographic modeling. Transportation Research Record: Journal of the Transportation Research Board, 1731(1), 15-22.

Gordon, R. (2010). Muni may reduce stops to increase speed, save cash. San Francisco Gate. Howe, A. (2011). Principles of bus service planning: Allan Howes Associates.

Ibeas, Á., Alonso, B., \& Sainz, O. (2010). Optimizing bus stop spacing in urban areas. Transportation research part E: logistics and transportation review, 46(3), 446-458.

Kehoe, O. (2004). Effects of bus stop consolidation on transit speed and reliability: A test case. (Master of Science), University of Washington.

Marchal, M. (2012). La STM paie-t-elle le juste prix pour les bus hybrides? Métro.

MTA. (2000). System-wide service standards: MTA New York City Transit \& MTA Bus Company.

SFMTA. (2010). Transit Effectiveness Project Implementation Strategy: San Francisco Municipal Transportation Agency.

Stewart, C., \& El-Geneidy, A. (2014). All Aboard at All Doors: Route Selection and Running Time Savings Estimation for Multi-scenario All-Door Bus Boarding. Paper presented the Transportation Research Board 93rd Annual Meeting.

Tétreault, P. R., \& El-Geneidy, A. M. (2010). Estimating bus run times for new limited-stop service using archived AVL and APC data. Transportation Research Part A: Policy and Practice, 44(6), 390-402.

Translink. (2012). Transit-oriented communities: A primer on key concepts.

TTC. (2014). Improving pedestrian safety and customer journey times: Transit stops. Toronto: Toronto Transit Commission. 
Wagner, Z., \& Bertini, R. (2014). Benefit-cost evaluation method for transit stop removal. Transportation Research Board(2415).

Walker, J. (2012). Human transit: How clearer thinking about public transit can enrich our communities and our lives. Washington, DC: Island Press.

WMATA. (2009). Design and placement of transit stops: Washington Metropolitan Area Transit Authority. 